\title{
An accurate algorithm of PMU-based wide area measurements for fault detection using positive-sequence voltage and unwrapped dynamic angles
}

\author{
Muhammad Qasim Khan ${ }^{a}$, Musse Mohamud Ahmed ${ }^{a}$, Ahmed M.A. Haidar ${ }^{\text {a,b," }}$ \\ ${ }^{a}$ Department of Electrical and Electronics Engineering, Faculty of Engineering, Universiti Malaysia Sarawak, 94300 Kota Samarahan, Sarawak, Malaysia \\ ${ }^{\mathrm{b}}$ School of Mechanical and Electrical Engineering, University of Southern Queensland, Australia
}

\section{A R T I C L E I N F O}

\section{Keywords:}

Non-recursive Discrete Fourier Transform

Positive-sequence voltage

Synchro-phasor

Transmission lines

Dynamic angles

Faulty zone classification

\begin{abstract}
A B S T R A C T
Modern power system requires advanced and intelligent sensors-based protection such as a Phasor Measurement Unit that can provide faster, accurate, and real-time data acquisition. The aim is to allow accurate action-based performance for analysts in monitoring the transmission lines so that rapid actions can be taken during abnormal circumstances before the blackout occurs. Among different algorithms, this study focuses on modelling the nonrecursive phasor estimation method in a power Simulink environment for a standard test system equipped with a developed algorithm to detect the fault zone. The algorithm includes an index for faulty bus classification based on the positive-sequence voltage measurements of the pre-fault and post-fault conditions, where the bus with a maximum differential percentage is identified as a faulted bus. An important differentiation of this work is that the proposed algorithm can coordinate with all phasor measurement units to accurately determine the faulty line using the index of unwrapped dynamic phase angles. Furthermore, the robustness of the indices is analyzed in the presence of sudden load change, measurement noise, and during nonlinear high-impedance faults. The performance of the comprehensive algorithm is investigated on the IEEE 9-bus and 39-bus standard test systems by applying different faults scenarios, considering several factors such as fault inception angles, line-fault resistance, ground-fault resistance. The comparative studies have shown that the proposed indices can play a significant role in segregating the fault and non-fault conditions, as they are needed to supervise the appropriate relays for enhancing the overall security of the power grid.
\end{abstract}

\section{Introduction}

The synchronized phasor measurement systems have turned up the proficiency of measurement and the protection zone selection in power systems. With such advanced technology, it is possible now to continuously monitor the health of the power system [1]. For more excellent reliability with high speed and efficiency, developed sensors throughout the Electrical Power System (EPS) are installed to detect faults by using different phasor estimation algorithms. In this context, Phasor Measurement Unit (PMU) reports in milliseconds, letting the EPS completely observable at similar instants, which benefits the system analysts to pinpoint initial faults before the blackout occurrence [2]. Hence, the Wide Area Measurement System (WAMS) is the most desirable system that can provide effective backup protection using an appropriate scheme to overcome the cascade tripping problems [3-9]. Since the traditional protection systems that utilize information based on singleend relay have lower efficiency [10-12], it is necessary to employ data from both ends of the transmission lines (TL) with data synchronization via WAMS.

At present, WAMS includes PMUs based on intelligent electronic devices incorporating data synchronized at both ends of TL $[13,14]$. To enhance the power system protection via PMUs, the accurate identification of faulty TL with dynamic security analysis of power system is significant $[14,15]$. Further, the manifestation of PMU's efficiency is an essential step towards protecting TL by applying the differential protection philosophy based on Kirchhoff Laws [16,17]. Ref [18] is among the first that developed the differential protection scheme for a 33-kV TL and substation using the numerical relays along with the Global Positioning System (GPS), which added synchronization to the stream of samples. The study based on traveling waves used to determine fault location was however adversely affected by source inductance and attenuation with the distance it travelled [19]. Traveling Wave Theory [20-22], and impedance-based method [23] techniques were analyzed using software simulations or by embedding the algorithms in hardware

\footnotetext{
* Corresponding author.

E-mail addresses: ahahmed@unimas.my, ahmed.haidar@usq.edu.au (A.M.A. Haidar).
} 